\title{
ANÁLISE DA PERDA DE VIDA DE TRANSFORMADORES DE POTÊNCIA USANDO SÉRIES TEMPORAIS E TEORIA DE CONFIABILIDADE
}

\author{
Maria Emilia Camargo e Luis Felipe Dias Lopes \\ Departamento de Estatística - Centro de Ciências Naturais e Exatas \\ UFSM - Santa Maria, RS
}

\section{RESUMO}

Neste trabalho aplicou-se as técnicas de Séries Temporais e Teoria de Confiabilidade para analisar a perda de vida de transformadores de potência, quando submetidos a uma sobrecarga. Foram usadas temperaturas diárias do enrolamento de um dos Transformadores de Potência de 100 MVA da Termoelétrica de Candióta, Candióta - RS. O período de análise está compreendido de dezembro de 1992 a outubro de 1993.

Palavras chaves: Séries Temporais, Teoria de Confiabilidade, Transformador de Potência, Perda de Vida

\begin{abstract}
In this paper, the time series methodology and reliability theory have been applied to analyze the loss of life of power transformers, when subjected to over load. The daily temperature of power transformer coil of 100 MVA collected from the Termoeletric Power Plant of Candiota, Candiota, RS have been used. The time period analyzed is from December, 1992 to October, 1993.
\end{abstract}

Key Words: Time Series, Reliability Theory, Power Transformer, Loss of Life. 


\section{1 - INTRODUÇÃo}

Devido à falta de controle nos grandes sistemas elétricos brasileiros, principalmente no planejamento de vida útil dos transformadores de potência, há uma necessidade de detectar o tempo de vida útil perdida (Bazovsky, 1961; Camargo, 1991). A necessidade de utilização de um transformador mais próximo de sua capacidade máxima, tem gerado aperfeiçoamentos nas normas e nos critérios que estabelecem os limites de carregamento dos transformadores (Dummer \& Griffin, 1970; Dummer \& Winton, 1974). Logo não devemos somente levar em conta os valores nominais de temperatura e potência, e sim reconhecer os conceitos de perda de vida. Este trabalho analisou a perda de vida através da análise da sobrecarga e a elevação excessiva da temperatura do enrolamento, de um transformador de potência de 100 MVA da Termoelétrica de Candióta - RS através da metodologia de Séries Temporais (Camargo, 1992) e Teoria de Confiabilidade Os dados foram coletados diariamente de dezembro de 1992 a outubro de 1993.

$\mathrm{O}$ artigo divide-se em quatro partes centrais, além desta introdução e da conclusão.

Na sessão 2, apresenta-se a Teoria sobre Transformadores de Potência. Na sessão 3, apresenta-se a Teoria de Confiabilidade. Na sessão 4 apresenta-se a Metodologia de Séries Temporais. Na sessão 5 apresenta-se Aplicą̧ão e Discussão e na sessão 6 encerra-se o artigo com as conclusões.

\section{2 - TRANSFORMADORES DE POTÊNCIA}

É um aparelho eletromagnético estático (sem peças móveis), por meio da qual a corrente alternada de uma tensão é transformada em corrente alternada de mesma freqüência, mas com outra tensão (FIGURA 1).

No transformador utilizamos um fenômeno chamado de self-indução (indução mútua), são dois enrolamentos atravessados por um fluxo magnético total.

\section{1 - Partes de um Transformador de Potência}

\subsection{1 - Núcleo}

É constituído de chapas de aço-silício, laminadas a frio, coberta de película isolante. ista lâmina seguida de um tratamento térmico, orienta os dominios magnéticos no sentido da ıminação., permitindo altas densidades de fluxo e baixas correntes de magnetização 


\subsection{2 - Enrolamentos}

São condutores enrolados em forma de bobinas cilindricas, com N1 e N2 espiras respectivamente, que são dispostas coaxialmente nas colunas do núcleo, em ordem crescente de tensão.

\subsection{3 - Isolamento}

É constituido basicamente de óleo e celulose (papel ou presnan), o óleo tem, ainda funções de resfriamento.

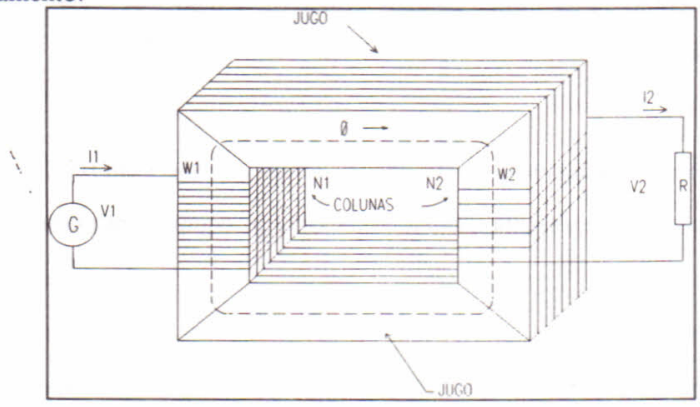

FIGURA 1 - Esquema de um Transformador de Potência

\subsection{4 - Aplicação de Cargas em Transformadores de Potência}

É ensaiado de acordo com a norma NBR 5256 da ABNT, para transformadores imersos em líquidos isolantes, e com potências nominais de 100 MVA. Esses procedimentos são baseados no emvelhecimento da isolação dos enrolamentos.

\section{2 - Cálculo da Perda de Vida de Transformadores sob Aplicação de Sobrecarga}

A equação básica para calcular a perda de vida percentual de transformadores de potência depende das seguintes variáveis

$\theta \mathbf{e}=$ temperatura do ponto mais quente do enrolamento;

$\Delta \mathrm{t}=$ intervalo de tempo genérico; (24)

$\mathrm{PV} \%$ = perda de vida percentual;

$A=-14,133$ (transformador de $55^{\circ} \mathrm{C}$ );

$A=-13,391$ (transformador de $65^{\circ} \mathrm{C}$ );

$\mathrm{B}=6972,15$;

PV\% $=10^{-\left(\frac{B}{273+\theta e}+A\right) \times 100 \times \Delta t}$ 


\section{3 - TEORIA DE CONFIABILIDADE}

Segundo Camargo (1991) a elaboração de principios que permitem o funcionamento de sistemas complexos, mesmo quando alguns de seus componentes estiverem defeituosos, é um dos objetivos da Teoria de Confiabilidade.

\section{1 - Tempo de vida de um componente (ou sistema)}

Seja então $F(t)$ um modelo para o tempo de vida de um determinado equipamento. A função

$R(t)=1-F(t), t \geq 0$

define a confiabilidade do equipamento para uma tarefa de duração $t$ em relação a função do tempo de vida. Observa-se que T representa a duração da vida do equipamento considerado, logo tem-se que

$R(t)=P(T>t), t \geq 0$

refere-se à probabilidade do equipamento cumprir interruptamente uma tarefa no prazo fixado ( $\mathrm{t}$ ).

\section{2 - Taxa de Falha}

Além da função $\mathrm{R}(\mathrm{t})$ de confiabilidade, temos outra função que desempenha importante papel na descrição das características de falha (ou avaria) de um equipamento

Seja $Z(t)$ a taxa de falhas (instantânea) algumas vezes denominada Função de Risco associada a variável aleatória $T$ dada por

$\mathrm{Z}(\mathrm{t})=\frac{\mathrm{f}(\mathrm{t})}{1-\mathrm{F}(\mathrm{t})}=\frac{\mathrm{f}(\mathrm{t})}{\mathrm{R}(\mathrm{t})}=\lambda_{\mathrm{t}}$

\section{3 - Estimação da Função de Confiabilidade}

Para estimar a Função de Confiabilidade, calcula-se inicialmente, uma estimativa para a Perda de Vida $\left(\lambda_{t}\right)$, que são organizadas em "Tabelas de Vida".

A probabilidade de que um equipamento, esteja em funcionamento num determinado período de tempo t, e falhar no próximo periodo de tempo é denotada por $\lambda_{t}$, e é chamado de Perda de Vida (taxa de falha), que vem a ser a probabilidade de que a falha ocorra no intervalo [t, $t+\Delta t$ ], dado que não ocorreu até o instante $t$.

$\lambda_{\mathrm{t}}=\mathrm{P}[\mathrm{t} \leq \mathrm{T} \leq \mathrm{t}+\Delta \mathrm{t} \forall \mathrm{T}>\mathrm{t}]$.

Uma vez obtidos os estimadores para $\lambda_{t}$, podem ser construídos estimadores para 


$$
\begin{aligned}
& \text { Para qualquer tempo, tem-se: } \\
& \hat{R}(t)=\prod_{i=1}^{t}\left[\left(1-\lambda_{t}\right)\left(\hat{R}(t)_{i-1}\right)\right]
\end{aligned}
$$

Este estimador é chamado de Função de Confiabilidade $\mathrm{R}(\mathrm{t})$, onde os periodos de tempo " $\mathrm{t}$ " são determinados pelos próprios dados e se constituem nos intervalos entre as Perdas de Vida.

\section{4 - METODOLOGIA DE SÉRIES TEMPORAIS}

$\mathrm{Na}$ análise estatística de uma série temporal, uma das metodologias, mais frequentemente utilizada é aquela proposta por Box \& Jenkins (1976), que utiliza uma ampla classe de modelos para representar processos estacionários e não estacionários homogêneos.

A estratégia de modelagem de Box \& Jenkins é composta por um ciclo iterativo de três etapas:

i) identificação de um ou mais modelos tentativos - as principais ferramentas usadas nessa etapa são as funções de autocorrelação e autocorrelação parcial;

ii) estimação e diagnóstico (para verificar a aleatoriedade dos resíduos);

iii) previsão com o modelo supostamente adequado.

Normalmente, antes de iniciarmos o ciclo iterativo algum tipo de transformação nos dados pode ser necessário, por exemplo, a transformação de Box \& Cox, com o objetivo principal de "simetrizar"os dados e estabilizar a variância.

Em sua formação geral, para possibilitar a representação de séries sazonais e/ou séries com não-estacionariedade homogênea (séries que se tornam estacionárias por aplicação de "d" diferenças simples e/ou "D" diferenças sazonais), os processos ARIMA multiplicativos são denotados por:

$$
\phi(B) \Phi\left(B^{s}\right)\left(1-B^{s}\right) D Z_{t}=\theta(B) \Theta\left(B^{s}\right) a_{t}
$$

onde:

$\mathrm{Z}_{\mathrm{t}} ; \mathrm{t}=1, \ldots, \mathrm{n} \Rightarrow$ é a série observada ;

$\mathrm{B} \Rightarrow$ é o operador de retardo, tal que $\mathrm{B}^{\mathrm{k}} \mathrm{Z}_{\mathrm{t}}=\mathrm{Z}_{\mathrm{t}-\mathrm{k}}$;

$\left\{\mathrm{a}_{\imath}\right\} \Rightarrow$ é uma seqüência de variáveis aleatórias independentes e identicamente distribuidas $\left(0, \sigma_{\mathrm{a}}^{2}\right)$, chamados de ruido branco;

$\Phi\left(B^{\mathrm{S}}\right)$ e $\Theta\left(B^{\mathrm{S}}\right) \Rightarrow$ são os polinômios sazonais de graus $\mathrm{P}$ e Q respectivamente em $\mathrm{B}$; 
$\Delta^{\mathrm{d}}=(1-\mathrm{B})^{\mathrm{d}}$ e $\Delta_{\mathrm{S}}^{\mathrm{D}}=\left(1-\mathrm{B}^{\mathrm{S}}\right)^{\mathrm{D}} \Rightarrow$ são os operadores de diferença simples sazonal, os quais formam o processo estacionário.

$\mathrm{S} \Rightarrow$ é o periodo sazonal.

Finalmente, deve-se salientar que as raizes dos polinômios devem cair fora do círculo unitário para garantir as usuais condições de estacionariedade e inversibilidade.

A ordem desse modelo é denotado por $(\mathrm{p}, \mathrm{d}, \mathrm{q}) *(\mathrm{P}, \mathrm{D}, \mathrm{Q})_{\mathrm{S}}$.

\section{5 - APLICAÇ̃̃o E DISCUSSÃo}

Aplicando-se a metodologia de Séries Temporais, o modelo que melhor se ajustou à série Temperatura do Enrolamento do Transformador de Potência foi um AR(1), com os seguintes parâmetros, apresentados na TABELA 1.

TABELA 1 - Parâmetros estimados e estatística " $t$ " para o modelo univariado com intervenção para a série Temperatura do Enrolamento

\begin{tabular}{l|c|c|c}
\hline \multicolumn{1}{c|}{ Parâmetro } & Lag & Coeficiente & "t" calculado \\
\hline Média & --- & 52.977 & 52.74 \\
Auto Regressivo & 1 & 0.6984 & 16.67 \\
Degrau $\left(\xi_{1}\right)$ & 164 & -1.3479 & -9.41 \\
Impulso $\left(\xi_{2}\right)$ & 83 & -1.1467 & -3.58 \\
Impulso $\left(\xi_{3}\right)$ & 131 & -1.1711 & -3.66 \\
Impulso $\left(\xi_{4}\right)$ & 48 & -1.0345 & -3.23 \\
Impulso $\left(\xi_{5}\right)$ & 210 & 1.0908 & 3.41 \\
\hline
\end{tabular}

As intervenções identificadas para a série de Temperatura do Enrolamento durante o período analisado, a um nivel de significância de 5\% estão apresentados na TABELA 2.

TABELA 2 - Tipos de intervenção detectadas

\begin{tabular}{c|c|c}
\hline \hline Tipo de Intervenção & Instante & Período \\
\hline $\mathrm{X}_{1 \mathrm{t}} \rightarrow 2$ & 164 & 13 de Maio \\
$\mathrm{X}_{2 \mathrm{t}} \rightarrow 1$ & 83 & 21 Fevereiro \\
$\mathrm{X}_{3 \mathrm{t}} \rightarrow 1$ & 131 & 10 Abril \\
$\mathrm{X}_{\mathrm{4t}} \rightarrow 1$ & 48 & 17 de Janeiro \\
$\mathrm{X}_{5 \mathrm{t}} \rightarrow 1$ & 210 & 28 de Junho \\
\hline
\end{tabular}

A representação do modelo univariado é da seguinte forma:

$$
\mathrm{Y}_{\mathrm{t}}=\mu+\phi_{1} \mathrm{Y}_{\mathrm{t}-1}+\xi_{1} \mathrm{X}_{1 \mathrm{t}}+\xi_{2} \mathrm{X}_{2 \mathrm{t}}+\xi_{3} \mathrm{X}_{3 \mathrm{t}}+\xi_{4} \mathrm{X}_{4 \mathrm{t}}+\xi_{5} \mathrm{X}_{5 \mathrm{t}}+\mathrm{a}_{\mathrm{t}}
$$




$$
\begin{aligned}
\mathrm{Y}_{\mathrm{t}}= & \underset{(52.74)}{52.977}+\underset{(16.67)}{0.6985 \mathrm{Y}_{\mathrm{t}-1}}-1.3 \underset{(-9.41)}{34} \mathrm{X}_{t}(164)-1.1 \underset{(-3.58)}{1487} \mathrm{X}_{t}(83)-\underset{(-3.23)}{1.1711} \mathrm{X}_{t}(131) \\
& -\underset{(-3.23)}{1.0345} \mathrm{X}_{t}(48)+\underset{(3.41)}{1.0908} \mathrm{X}_{t}(210)+\mathrm{a}_{\mathrm{t}}
\end{aligned}
$$

As estatísticas de ajuste para o modelo são:

$\mathrm{R}^{2}=0.8036$

AIC $=-1.8569$

As estatisticas do ruido são:

Média $=-0.000011494 \cong 0.00$

Variância $=0.149011$

Após ter encontrado o melhor modelo foi feita uma previsão de temperatura do enrolamento para os demais 65 dias a frente, a fim de testar a robustez do modelo em relação a previsão, bem como completar um ano.

\section{6 - CONCLUSÃo}

Para a série original (300 dias) foi construido um algoritmo em DBASE para determinar a perda de vida para esse periodo $(\mathrm{PV}=0.0311156)$ e para os demais dias previstos obtendo-se uma perda de vida de 0.0009194 , logo conclui-se que para 1 ano de funcionamento a perda de vida é de 0.032035 , ou seja $3.2 \%$ ao ano. Levando-se em conta que a temperatura média do enrolamento para este periodo foi de $45.2^{\circ} \mathrm{C}$.

Partindo-se deste principio e admitindo-se que este transformador vem sendo submetido a mesma sobrecarga desde 1973 (20 anos) pode-se concluir que a perda de vida para esses periodo fica em torno de $51.87 \%$, e que o mesmo tem um tempo de vida de aproximadamente 19 anos.

\section{5 - REFERÊNCIAS BIBLIOGRÁFICAS}

BAZOVSKY, I. (1961). Reliability theory and practice. New Jersey: Prentice-Hall.

CAMARGO. C.C.B. (1991). Confiabilidade aplicada a sistemas de potência elétrica. Rio de Janeiro: Livro Técnico e Científico.

CAMARGO, M.E. (1992). Modelagem Clássica e Bayesiana: uma evidência empírica do processo inflacionário brasileiro. Programa de Pós-Graduação em Engenharia de Produção, UFSC. (Tese de Doutorado). 
DUMMER, G.W.A., GRIFFIN, N.B. (1970). Eletronics reliability calculation and desing. Oxford: Pergamon Press

DUMMER, G.W.A., WINTON, R.C. (1974) An elementary guide to reliability. New York: Pergamon Press 